Article

\title{
Sodium Thiosulfate Improves Hypertension in Rats with Adenine-Induced Chronic Kidney Disease
}

\author{
Chien-Ning Hsu ${ }^{1,2}\left(\mathbb{D}\right.$, Chih-Yao Hou ${ }^{3}(0)$, Guo-Ping Chang-Chien $\left.{ }^{4,5}{ }^{(}\right)$, Sufan Lin ${ }^{4,5}$, Hung-Wei Yang ${ }^{6, *(1)}$ \\ and You-Lin Tain $7,8, *$ (D)
}

Citation: Hsu, C.-N.; Hou, C.-Y.; Chang-Chien, G.-P.; Lin, S.; Yang, H.-W.; Tain, Y.-L. Sodium Thiosulfate Improves Hypertension in Rats with Adenine-Induced Chronic Kidney Disease. Antioxidants 2022, 11, 147. https://doi.org/10.3390/antiox11010147 Academic Editors: Emma Mitidieri and Vincenzo Brancaleone

Received: 25 November 2021

Accepted: 10 January 2022

Published: 11 January 2022

Publisher's Note: MDPI stays neutral with regard to jurisdictional claims in published maps and institutional affiliations.

Copyright: (c) 2022 by the authors. Licensee MDPI, Basel, Switzerland. This article is an open access article distributed under the terms and conditions of the Creative Commons Attribution (CC BY) license (https:// creativecommons.org/licenses/by/ $4.0 /)$.
1 Department of Pharmacy, Kaohsiung Chang Gung Memorial Hospital, Kaohsiung 833, Taiwan; cnhsu@cgmh.org.tw

2 School of Pharmacy, Kaohsiung Medical University, Kaohsiung 807, Taiwan

3 Department of Seafood Science, National Kaohsiung University of Science and Technology, Kaohsiung 811, Taiwan; chihyaohou@webmail.nkmu.edu.tw

4 Center for Environmental Toxin and Emerging-Contaminant Research, Cheng Shiu University, Kaohsiung 833, Taiwan; guoping@csu.edu.tw (G.-P.C.-C.); linsufan2003@csu.edu.tw (S.L.)

5 Super Micro Mass Research and Technology Center, Cheng Shiu University, Kaohsiung 833, Taiwan

6 Institute of Medical Science and Technology, National Sun Yat-sen University, Kaohsiung 804, Taiwan

7 Department of Pediatrics, Kaohsiung Chang Gung Memorial Hospital and Chang Gung University College of Medicine, Kaohsiung 833, Taiwan

8 Institute for Translational Research in Biomedicine, Kaohsiung Chang Gung Memorial Hospital and Chang Gung University College of Medicine, Kaohsiung 833, Taiwan

* Correspondence: howardyang@imst.nsysu.edu.tw (H.-W.Y.); tainyl@hotmail.com (Y.-L.T.)

\begin{abstract}
Hypertension is highly prevalent in chronic kidney disease (CKD). Hydrogen sulfide $\left(\mathrm{H}_{2} \mathrm{~S}\right)$ is an endogenously produced gasotransmitter with vasodilator properties. We, hence, investigated whether oral administration of sodium thiosulfate (STS), a clinically applicable $\mathrm{H}_{2} \mathrm{~S}$-based therapy, can exert a protective effect against hypertension in an adenine-induced CKD rat model. Eightweek-old male Sprague-Dawley rats were fed with $0.5 \%$ adenine chow for 3 weeks to induce CKD. After 1 week, the rats were divided into two groups: one without and one with STS ( $2 \mathrm{~g} / \mathrm{kg}$ body weight/day) in drinking water for 2 weeks. Treatment with STS lowered systolic and diastolic blood pressure by 7 and $9 \mathrm{~mm} \mathrm{Hg}$, respectively. Renal $\mathrm{H}_{2} \mathrm{~S}$-generating enzyme expression was inhibited by CKD, while STS therapy increased plasma levels of $\mathrm{H}_{2} \mathrm{~S}$ and thiosulfate. Additionally, restoration of nitric oxide bioavailability and rebalance of the renin-angiotensin system may contribute to the protective effects of STS. Our data suggest that the oral administration of STS improves hypertension in an adenine-induced CKD model, which brings us closer to the clinical translation of $\mathrm{H}_{2} \mathrm{~S}$-targeting therapy in CKD-induced hypertension.
\end{abstract}

Keywords: symmetric dimethylarginine; chronic kidney disease; hydrogen sulfide; hypertension; nitric oxide; renin-angiotensin system; sodium thiosulfate

\section{Introduction}

Hydrogen sulfide $\left(\mathrm{H}_{2} \mathrm{~S}\right)$ is a gasotransmitter, which plays an important role in several biological functions, including the renal regulation of blood pressure (BP) [1,2]. Hypertension and chronic kidney disease (CKD) both are common diseases all over the world [3], being closely connected to each other. Emerging evidence suggests that a reduction in $\mathrm{H}_{2} \mathrm{~S}$ synthesis may be a key mechanism underlying hypertension and kidney disease $[1,2,4]$. In the kidneys, a mismatch of vasodilators, such as $\mathrm{H}_{2} \mathrm{~S}$ and nitric oxide (NO), and vasoconstrictors, such as angiotensin II (Ang II), in favor of the latter, may ultimately lead to hypertension $[5,6]$. Conversely, $\mathrm{H}_{2} \mathrm{~S}$-based modalities have been developed for therapeutic protection against hypertension and kidney disease [6,7].

Enzymatic production of $\mathrm{H}_{2} \mathrm{~S}$ from the substrate L-Cysteine occurs through three enzymes: cystathionine-lyase (CSE), cystathionine-synthase (CBS), and 3-mercaptopyruvate 
sulfurtransferase (3MST) [1]. In an alternative pathway, $\mathrm{H}_{2} \mathrm{~S}$ is produced by cysteine amino transferase (CAT) and D-amino acid oxidase (DAO). Our prior work showed that dietary supplementation with L-Cysteine (the precursor of $\mathrm{H}_{2} \mathrm{~S}$ ) protects spontaneously hypertensive rats (SHRs) against high-salt-induced hypertension and kidney damage via the reduction of oxidative stress, mediation of the $\mathrm{H}_{2} \mathrm{~S}$-generating pathway, and restoration of the renin-angiotensin system (RAS) [8].

In addition, $\mathrm{H}_{2} \mathrm{~S}$ can be non-enzymatically produced from organic thiol. Thiosulfate, an intermediate in oxidative $\mathrm{H}_{2} \mathrm{~S}$ metabolism, can alternatively be reduced and regenerate $\mathrm{H}_{2} \mathrm{~S}$ [9]. Although thiosulfate has shown therapeutic potential in hypertensive renal disease in a model of NO deficiency [10], limited information is available about whether thiosulfate therapy can protect against hypertension in CKD.

The adenine diet model results in progressive kidney injury and several complications, truly mimicking the pathophysiology of human CKD [11]. Our prior work illustrated that rats receiving chow supplemented with $0.5 \%$ adenine for 3 weeks develop CKD, characterized as renal dysfunction, glomerular and tubulointerstitial damage, hypertension, and increased uremic toxin levels [12]. The objective of this study reported here was to examine whether sodium thiosulfate (STS) treatment can protect against CKD-induced hypertension and elucidate the underlying mechanism using an adenine-induced CKD model.

\section{Materials and Methods}

\subsection{Animal Model and Care}

Male Sprague-Dawley (SD) rats $(n=32)$ were obtained from BioLASCO Taiwan Co., Ltd. (Taipei, Taiwan). On arrival, the rats were maintained in our animal facility awarded full accreditation from AAALAC International. The procedures used in this study were according to the rules of the Care and Use of Laboratory Animals of the National Institutes of Health and the IACUC of Chang Gung Memorial Hospital (Permit \# 2020073101).

Afterward, each rat was randomly assigned to one of four treatments ( $n=8$ per group): normal diet (ND), diet supplemented with $0.5 \%$ adenine from 8 weeks of age for 3 weeks (CKD), normal diet with STS $(2 \mathrm{~g} / \mathrm{kg}$ body weight/day) in drinking water from 9 weeks of age for 2 weeks (ND + STS), and diet supplemented with $0.5 \%$ adenine from weeks 8 to 11 and STS from weeks 9 to 11 (CKD + STS). The doses of adenine and STS used here are based on previous studies conducted on rats [10,11]. We used the CODA rat tail-cuff system (Kent Scientific Corporation, Torrington, CT, USA) to measure BP [12]. All rats were acclimated to restraint and tail-cuff inflation for 1 week before the initiation of the experiment to ensure reproducibility and accuracy. All rats were sacrificed at 11 weeks of age. Blood samples were collected in tubes containing heparin. The kidneys were immediately snap-frozen and saved at $-80^{\circ} \mathrm{C}$ until analysis.

\subsection{Analysis of Plasma Hydrogen Sulfide and Thiosulfate Levels}

According to our validated protocol [13], concentrations of $\mathrm{H}_{2} \mathrm{~S}$ and thiosulfate in the plasma were determined by an Agilent Technologies 1290 high-performance liquid chromatography (HPLC) system connected to an Agilent 6470 Triple Quadrupole LC/Mass Spectrometry (MS) (Agilent Technologies, Wilmington, DE, USA). We added internal standard phenyl 4-hydroxybenzoate (PHB) to the extraction solvent. We detected thiosulfate derivative pentafluorobenzyl (PFB) $-\mathrm{S}_{2} \mathrm{O}_{3} \mathrm{H}$ and $\mathrm{H}_{2} \mathrm{~S}$ derivative sulfide dibimane (SDB). Selected reaction monitoring mode was used to detect target compounds with a targeted $\mathrm{m} / \mathrm{z}$ $212.99 \rightarrow 93, m / z 415 \rightarrow 223$, and $m / z 292.99 \rightarrow 81$ for $\mathrm{PHB}$, SDB, and PFB- $\mathrm{S}_{2} \mathrm{O}_{3} \mathrm{H}$, respectively.

\subsection{Quantitative Real-Time Polymerase Chain Reaction ( $q P C R$ )}

Rat kidney cortex tissue was homogenized in lysis buffer, and total RNA was extracted using the TRIZOL method (Invitrogen, Carlsbad, CA, USA), as described earlier [13]. Twostep quantitative real-time PCR was conducted in duplicate using the QuantiTect SYBR Green PCR Kit (Qiagen, Valencia, CA, USA) on an iCycler iQ Real-Time PCR Detection 
System (Bio-Rad, Hercules, CA, USA). A total of four genes involved in $\mathrm{H}_{2} \mathrm{~S}$ production were determined: Cbs (encoding for protein CBS), Cth (CSE), Mpst (3MST), and Dao (DAO). We also determined the renal expression of the genes belonging to the RAS, including Ren (renin), Ace (angiotensin converting enzyme-1), Agtr1a (angiotensin II type 1 receptor), Ace 2 (angiotensin converting enzyme-2), Agtr2 (angiotensin II type 2 receptor), and Mas1 (angiotensin (1-7) receptor MAS). Rn18s was used as the internal control as it was expressed at a constant level across the sample set. The comparative threshold cycle (Ct) method $\left(2^{-\Delta \Delta C t}\right)$, represented as $x$-fold expression, was used to compare the mRNA levels. Table 1 provides the PCR primer sequences.

Table 1. PCR primer sequences.

\begin{tabular}{|c|c|c|}
\hline Gene & Forward $\left(5^{\prime}-3^{\prime}\right)$ & Reverse $\left(5^{\prime}-3^{\prime}\right)$ \\
\hline Cbs & atgctgcagaaaggcttcat & gtggaaaccagtcggtgtct \\
\hline Cth & cgcacaaattgtccacaaac & gctctgtccttctcaggcac \\
\hline Mpst & ggctcagtaaacatcccattc & tgtccttcacagggtcttcc \\
\hline Dao & ccctttctggaaaagcacag & ctcctctcaccacctcttcg \\
\hline Ren & aacattaccagggcaactttcact & accccettcatggtgatctg \\
\hline Ace & caccggcaaggtctgctt & cttggcatagtttcgtgaggaa \\
\hline Ace2 & accettcttacatcagccctactg & tgtccaaaacctaccccacatat \\
\hline Agtr1a & gctgggcaacgagtttgtct & cagtccttcagctggatcttca \\
\hline Agtr1b & caatctggctgtggctgactt & tgcacatcacaggtccaaaga \\
\hline Mas & catctctcctctcggctttgtg & cctcatccggaagcaaagg \\
\hline $\operatorname{Rn} 18 s$ & gccgcggtaattccagctcca & cccgccegctccaagatc \\
\hline
\end{tabular}

\subsection{Western Blot}

As previously described [8], kidney cortex samples were subjected to Western blotting with antibody incubation. For each sample, equal quantities of total protein $(200 \mu \mathrm{g})$ were loaded and electrophoresed through a $10 \%$ polyacrylamide gel. Separated proteins were transferred into a nitrocellulose membrane (GE Healthcare Bio-Sciences Corp., Piscataway, NJ, USA). We used Ponceau S staining (PonS) to correct for variations in the total protein loading in the Western blot. After transfer, the nitrocellulose membranes were rinsed briefly in distilled water and incubated in $15 \mathrm{~mL}$ of a Ponceau S solution $(0.2 \%(w / v)$ in $1 \%(v / v)$ acetic acid; Sigma-Aldrich, St. Louis, MO, USA) for $10 \mathrm{~min}$ on the rocker, followed by a brief rinse in distilled water so that the lanes and bands were clearly visible. The membranes were then scanned and the TIFF file saved for later quantification. After that, the membranes were incubated in distilled water for two washes of $5 \mathrm{~min}$ each and we proceeded with the blocking. The membranes were blocked in phosphate-buffered saline-Tween (PBS-T) containing 5\% nonfat milk. For the detection of $\mathrm{H}_{2} \mathrm{~S}$-generating enzymes, the membranes were incubated with the following antibodies: a rabbit monoclonal anti-rat 3MST antibody (1:500, overnight incubation; Novus Biologicals, Littleton, CO, USA), a mouse monoclonal anti-rat CBS antibody (1:1000, overnight incubation; Abnova Corporation, Taipei, Taiwan), or a rabbit polyclonal anti-rat CSE antibody (1:1000, overnight incubation; Proteintech Group, Inc. Chicago, IL, USA). The bands were detected using an enhanced chemiluminescence reagent (PerkinElmer, Waltham, MA, USA). The abundance of protein expression was quantified by densitometry (Quantity One Analysis software, Bio-Rad) and calculated as the integrated optical density (IOD) in relation to the background value. The relative protein abundance was expressed as IOD/PonS to correct the variations in total protein loading.

\subsection{Determination of Nitric Oxide Parameters}

We used the HP Agilent 1100 HPLC System (Agilent Technologies Inc., Santa Clara, CA, USA) with fluorescence detection of O-phthalaldehyde/3-mercaptopropionic acid (OPA/3-MPA) derivatives to measure NO-related parameters in the plasma. These parameters include L-Arginine, L-Citrulline (the precursor of L-Arginine), and NO synthase 
inhibitor asymmetric and symmetric dimethylarginine (ADMA and SDMA). The standards contained 1-100 mM L-Citrulline, 1-100 mM L-Arginine, 0.5-5 mM ADMA, and 0.5-5 mM SDMA. L-Arginine was divided by ADMA plasma levels for calculating the L-Arginine-to-ADMA ratio, a determinant of NO bioavailability [14].

\subsection{Statistical Analysis}

All data are presented as the mean \pm the standard error of the mean. Statistical analyses were performed using one-way ANOVA or two-way ANOVA where appropriate. Tukey's post hoc test was applied where multiple comparisons were made. $p<0.05$ was considered significant. Statistical analyses were performed using SPSS software (SPSS Inc., Chicago, IL, USA).

\section{Results}

\subsection{Body Weight and Blood Pressure of Male Offspring}

The body weight (BW) of the CKD and CKD + STS groups was lower, while the kidney weight $(\mathrm{KW})$ and the $\mathrm{KW}$-to-BW ratio were higher compared to those of the control (Table 2). Baseline BP values were not significantly different among groups. At 11 weeks of age, the CKD group showed increases in systolic and diastolic BP and the mean arterial pressure was significantly attenuated in the CKD + STS group. Taken together, these findings indicate that CKD-induced hypertension is improved by STS treatment.

Table 2. Weight and blood pressure.

\begin{tabular}{ccccc}
\hline Groups & ND & CKD & ND + STS & CKD + STS \\
\hline Body weight (BW) (g) & $374 \pm 6$ & $292 \pm 7 *$ & $377 \pm 7$ & $270 \pm 12 *$ \\
Left kidney weight (g) & $1.89 \pm 0.09$ & $3.54 \pm 0.23 *$ & $1.8 \pm 0.07$ & $2.94 \pm 0.17 *$ \\
Left kidney weight/100 g BW & $0.5 \pm 0.006$ & $1.21 \pm 0.060 *$ & $0.48 \pm 0.019$ & $1.09 \pm 0.037 *$ \\
Systolic BP (mmHg) & $129 \pm 1$ & $145 \pm 2 *$ & $132 \pm 1$ & $138 \pm 1 \#$ \\
Diastolic BP (mmHg) & $86 \pm 2$ & $101 \pm 3 *$ & $90 \pm 3$ & $92 \pm 2 \#$ \\
Mean arterial pressure (mmHg) & $100 \pm 1$ & $115 \pm 2 *$ & $104 \pm 2$ & $107 \pm 1 \#$ \\
\hline$n=8$ /group; ${ }^{*} p<0.05$ vs. ND; $p<0.05$ CKD + STS vs. CKD; BP = blood pressure. &
\end{tabular}

\section{2. $\mathrm{H}_{2} \mathrm{~S}$ Pathway}

Results for the $\mathrm{H}_{2} \mathrm{~S}$ pathway are shown in Figure 1, which include plasma $\mathrm{H}_{2} \mathrm{~S}$ and thiosulfate levels and the renal mRNA expression of $\mathrm{H}_{2} \mathrm{~S}$-generating enzymes. Compared to $\mathrm{ND}$, the plasma $\mathrm{H}_{2} \mathrm{~S}$ level was higher in the other three groups (Figure $1 \mathrm{~A}$ ). The STS treatment showed a further increased $\mathrm{H}_{2} \mathrm{~S}$ level in the CKD + STS group compared to the CKD group. Similarly, an increased plasma thiosulfate level was observed in the ND + STS and CKD + STS groups compared to the control (Figure 1B). Renal transcript abundance of $\mathrm{H}_{2} \mathrm{~S}$-generating enzymes $\mathrm{Cbs}, \mathrm{Cth}, \mathrm{Dao}$, and Mpst is compared in Figure 1C. Compared to $\mathrm{ND}$, four $\mathrm{H}_{2} \mathrm{~S}$-generating enzymes showed similar downregulation patterns in the CKD and CKD + STS groups.

Renal protein abundance of $\mathrm{H}_{2} \mathrm{~S}$-generating enzymes is compared in Figure 2. CKD significantly reduced renal CBS and 3MST protein levels in the CKD and CKD + STS groups (Figure 2B,D), while STS treatment had a negligible effect on their levels. Although CKD showed a similar tendency to the renal CSE protein abundance, it did not reach the significance (Figure 2C). Collectively, these findings indicate that STS treatment increases plasma $\mathrm{H}_{2} \mathrm{~S}$ and thiosulfate levels without affecting renal $\mathrm{H}_{2} \mathrm{~S}$-generating enzyme expression. 

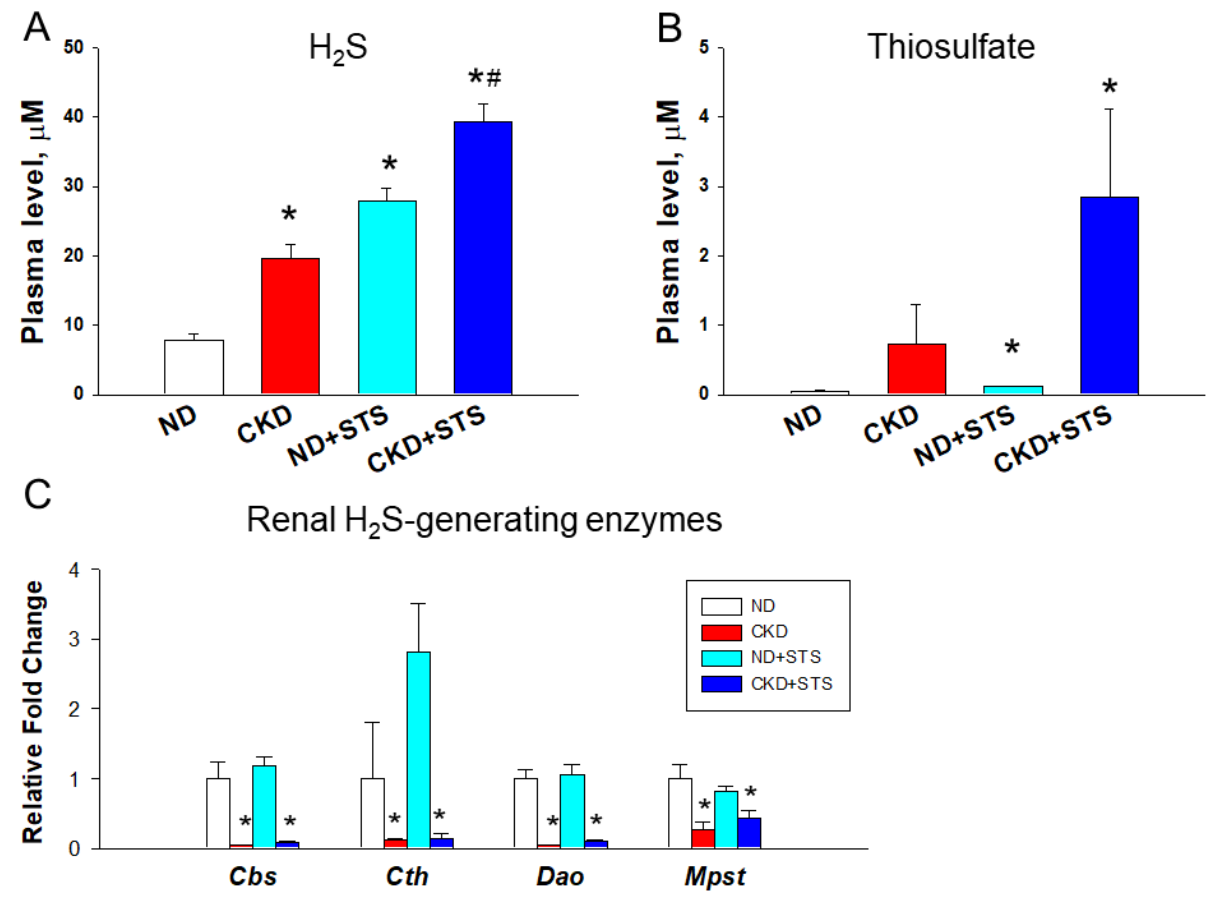

Figure 1. Effects of sodium thiosulfate (STS) on the (A) plasma $\mathrm{H}_{2} \mathrm{~S}$ level, (B) plasma thiosulfate level, and $(\mathbf{C})$ renal mRNA expression of $\mathrm{H}_{2} \mathrm{~S}$-generating enzymes. All the results represent the mean \pm the standard errors of eight animals in each group. Data are analyzed by one-way ANOVA followed by Tukey's post hoc test. ${ }^{*} p<0.05$ versus control g; ${ }^{*} p<0.05$ vs. ND; $\# p<0.05$ CKD.

A
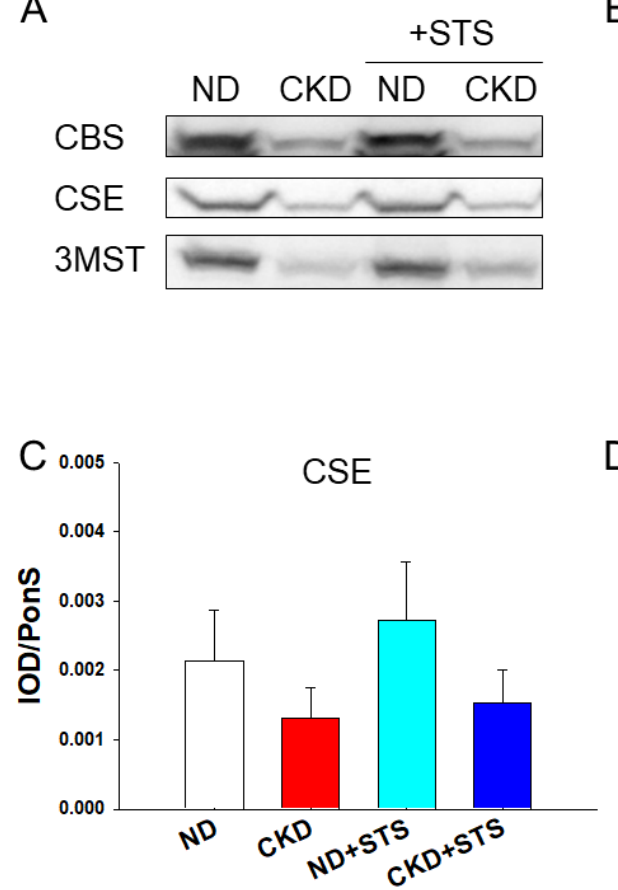

B
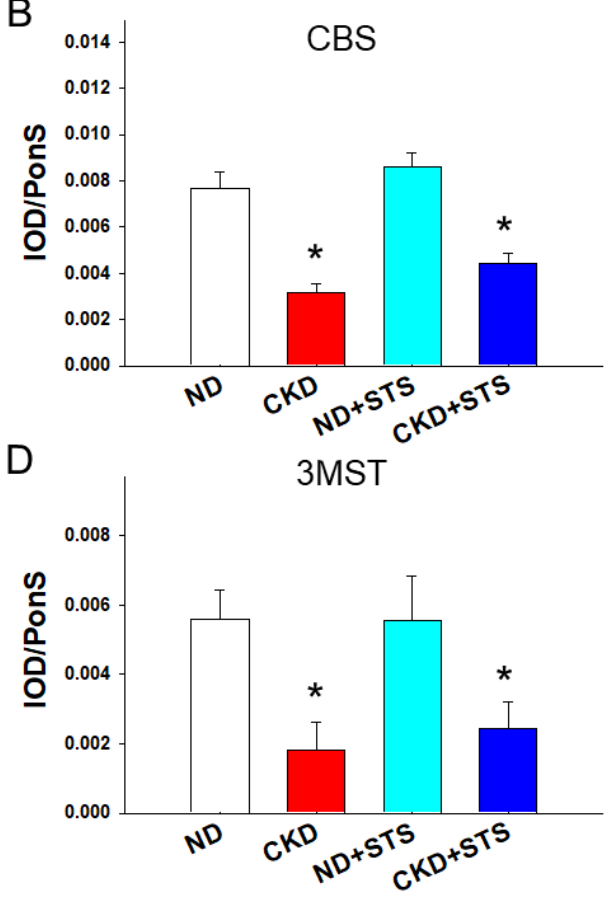

Figure 2. Effects of sodium thiosulfate (STS) on $\mathrm{H}_{2}$ S-generating enzymes in the kidneys. (A) Representative Western blots show cystathionine $\beta$-synthase (CBS, $\sim 61 \mathrm{kDa}$ ), cystathionine $\gamma$-lyase (CSE, $\sim 45 \mathrm{kDa}$ ), and 3-mercaptopyruvate sulfurtransferase (3MST, $\sim 52 \mathrm{kDa}$ ) bands. The relative abundance of renal cortical (B) CBS, (C) CSE, and (D) 3MST was quantified. All the results represent the mean \pm the standard errors of eight animals in each group. Data are analyzed by one-way ANOVA followed by Tukey's post hoc test. ${ }^{*} p<0.05$ vs. ND. 


\subsection{NO Pathway}

Plasma NO parameters are compared in Table 3. Compared to ND, plasma L-Citrulline and L-Arginine levels were lower in the other three groups. The CKD group had higher plasma ADMA levels, but thiosulfate reduced this effect. The STS treatment reduced SDMA in the ND + STS group. In addition, CKD only reduced the L-Arginine-to-ADMA ratio in rats with $C K D$. These results therefore indicate that in rats with $C K D$, the NO pathway is impaired, characterized as increased ADMA but decreased L-Arginine and decreased L-Arginine-to-ADMA ratio. In contrast, decreased NO bioavailability was improved by STS therapy.

Table 3. Plasma NO parameters.

\begin{tabular}{ccccc}
\hline Groups & ND & CKD & ND + STS & CKD + STS \\
\hline L-Citrulline $(\mu \mathrm{M})$ & $117.3 \pm 7$ & $101.3 \pm 5.3^{*}$ & $83.8 \pm 6.8^{*}$ & $99.1 \pm 3.9^{*}$ \\
L-Arginine $(\mu \mathrm{M})$ & $333.2 \pm 15.5$ & $236.8 \pm 11.2^{*}$ & $231.7 \pm 28.4^{*}$ & $190.1 \pm 17.8^{* \#}$ \\
Asymmetric dimethylarginine $(\mu \mathrm{M})$ & $1.51 \pm 0.17$ & $1.96 \pm 0.12^{*}$ & $1.01 \pm 0.18$ & $1.21 \pm 0.09 \#$ \\
Symmetric dimethylarginine $(\mu \mathrm{M})$ & $1.18 \pm 0.07$ & $1.21 \pm 0.13$ & $0.81 \pm 0.09^{*}$ & $1.07 \pm 0.18$ \\
L-Arginine-to-ADMA ratio $(\mu \mathrm{M} / \mu \mathrm{M})$ & $242 \pm 30$ & $125 \pm 11^{*}$ & $254 \pm 29$ & $167 \pm 23$ \\
\hline
\end{tabular}

All the results represent the mean \pm the standard errors of eight animals in each group. Data are analyzed by one-way ANOVA followed by Tukey's post hoc test. ${ }^{*} p<0.05$ vs. ND; \# $p<0.05$ vs. CKD.

\subsection{Renin-Angiotensin System}

We further evaluated the RAS genes by qPCR (Figure 3). CKD reduced the renal mRNA expression of Ace2, which was restored by STS treatment. The STS treatment significantly reduced CKD-induced increases of renal Agtr1a expression. Additionally, Mas1 expression was greater in the CKD + STS group compared with that in the CN and CKD groups.

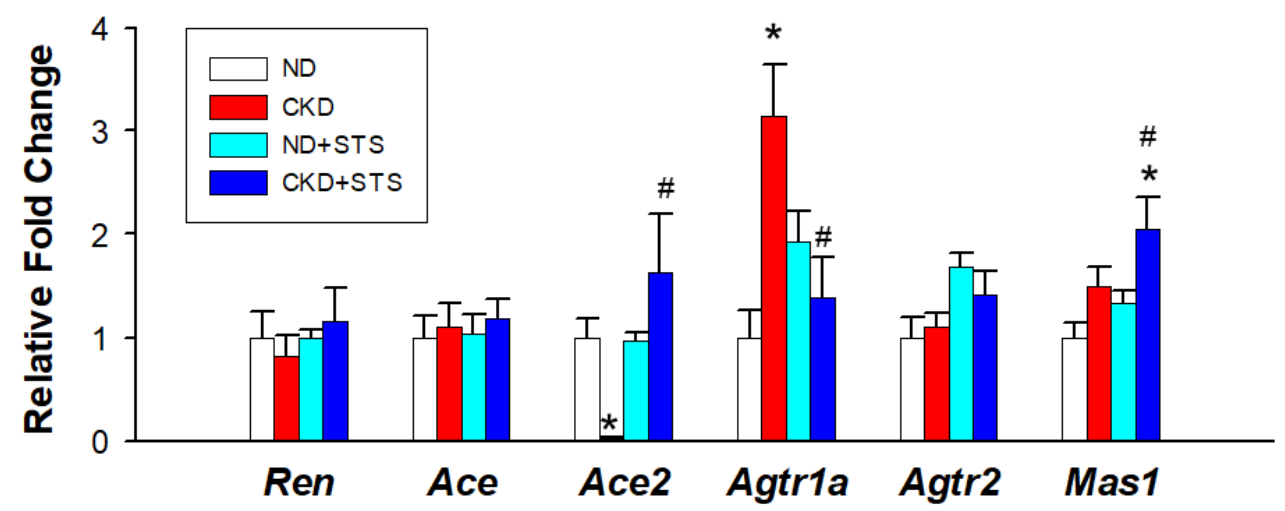

Figure 3. Effects of sodium thiosulfate (STS) on the renin-angiotensin system. All the results represent the mean \pm the standard errors of eight animals in each group. Data are analyzed by one-way ANOVA followed by Tukey's post hoc test. ${ }^{*} p<0.05$ vs. ND; \# $p<0.05$ vs. CKD.

\section{Discussion}

This work casts new light on the interaction between $\mathrm{H}_{2} \mathrm{~S}$ signaling, the $\mathrm{NO}$ pathway, and the RAS in the kidney by which STS therapy prevents hypertension induced by CKD. The major contributions of our study are summarized as follows: (1) STS treatment attenuated the elevation of BP in rats with adenine-induced CKD, (2) CKD caused a reduction in renal $\mathrm{H}_{2} \mathrm{~S}$-generating enzyme expression, while STS treatment had a negligible effect on their levels, (3) STS therapy increased plasma levels of $\mathrm{H}_{2} \mathrm{~S}$ and thiosulfate, (4) the beneficial effect of STS was associated with the reduction of ADMA and the restoration of L-Arginine-to-ADMA ratio in the plasma, and (5) STS treatment enhanced RAS gene Ace2 and Mas1 but decreased Agtr1a. 
The findings of this research are in line with prior studies indicating that decreased renal $\mathrm{H}_{2} \mathrm{~S}$-generating enzyme protein levels and/or activity is involved in the pathogenesis of hypertension and CKD $[15,16]$. Although $\mathrm{H}_{2} \mathrm{~S}$ exhibits a wide range of biological functions [1,2,17], limited data are available regarding the effects of STS on CKD-induced hypertension. According to our data, treatment with STS increased plasma levels of $\mathrm{H}_{2} \mathrm{~S}$ and thiosulfate while it had little effect on renal $\mathrm{H}_{2} \mathrm{~S}$-generating enzymes.

The reduction in BP observed in this study is consistent with previous findings displaying the vasorelaxant properties of $\mathrm{H}_{2} \mathrm{~S}[7,8,13]$. $\mathrm{H}_{2} \mathrm{~S}$ can be endogenously produced or exogenously delivered. We have previously shown that the administration of precursors of $\mathrm{H}_{2} \mathrm{~S}$, such as L-Cysteine or $\mathrm{N}$-Acetylcysteine, could enhance endogenous $\mathrm{H}_{2} \mathrm{~S}$ production and provide protection against hypertension $[8,18]$. Considering $\mathrm{H}_{2} \mathrm{~S}$-generating enzymes are inhibited by CKD in our model, this might not be an appropriate approach for CKDinduced hypertension. $\mathrm{H}_{2} \mathrm{~S}$ donors have been evaluated as having the therapeutic potential of exogenous $\mathrm{H}_{2} \mathrm{~S}$ [19]. However, exogenous $\mathrm{H}_{2} \mathrm{~S}$ donors might be toxic whenever they cause a short-term increase in $\mathrm{H}_{2} \mathrm{~S}$ to supraphysiological concentrations, which limit their clinical translation.

The current study provides another possibility in the use of sodium thiosulfate. As dynamic conversion exists between thiosulfate and $\mathrm{H}_{2} \mathrm{~S}$ [9], thiosulfate can serve as a steady source of $\mathrm{H}_{2} \mathrm{~S}$ in our body. Treatment with STS for 2 weeks in our study reduced SBP and DBP by 7 and $9 \mathrm{mmHg}$ in rats with adenine-induced CKD, respectively. The BP lowering effect of STS in our work is similar to that previously reported in an NO deficiency model [10]. In the present study, the antihypertensive effects of STS were achieved in the face of increases in plasma thiosulfate and $\mathrm{H}_{2} \mathrm{~S}$. As STS has been used for the treatment of calciphylaxis and has a proven safety profile [20], our data have extended the possibility of using STS for CKD-induced hypertension for future clinical translation.

A key vasorelaxant action of $\mathrm{H}_{2} \mathrm{~S}$ is to enhance $\mathrm{NO}$ signaling $[1,17]$. In our CKD model, the beneficial effect of STS is likely considering that it relies on the restoration of $\mathrm{NO}$ bioavailability. In the current study, the CKD-caused elevation of BP coincides with an increased ADMA level and a decreased ratio of L-Arginine to ADMA, a determinant of NO bioavailability. Conversely, these changes were restored by STS treatment. Accordingly, the protective effect of STS against hypertension is, at least in part, attributed to its ability to restore reduced NO bioavailability. However, the extent to which the organ-specific expression of NO synthase enzymes was affected by STS awaits further evaluation. Notably, previous studies have revealed cross-talk mechanisms between $\mathrm{H}_{2} \mathrm{~S}, \mathrm{NO}$, and carbon monoxide (CO) against hypertension $[4,6] . \mathrm{H}_{2} \mathrm{~S}$ can modify cysteine residues on key signaling molecules such as nuclear factor erythroid 2-related factor 2 (NRF2) and nuclear factor $\kappa \mathrm{B}(\mathrm{NF} \kappa \mathrm{B})$, thereby promoting antioxidant and anti-inflammatory effects [6]. Activation of the NRF2-heme oxygenase-1 (HO-1)-CO signaling pathway can counterbalance oxidative stress [21]. NO can mediate NFKB to reduce inflammation [22]. Because of the cross-talk between the three gasotransmitters, there will be a growing need to better understand the possible contribution of $\mathrm{NO}$ and $\mathrm{CO}$ production to the observed beneficial effects of STS.

Another probable beneficial effect of STS is related to its ability to rebalance the RAS. Consistent with prior research indicating that the aberrant activation of the classic RAS is associated with CKD [23], our data demonstrate that CKD enhances renal AT1R expression. It is well known that the classical RAS can be activated by Ang II to trigger kidney damage and vasoconstriction via AT1R [24]. Reduction of AT1R expression by STS therapy may offset the detrimental effects of Ang II signaling in favor of vasodilatation. Conversely, STS treatment enhances ACE2 and MAS expression. Activation of the counter-regulatory ACE2-angiotensin (1-7)-MAS receptor pathway is able to induce vasodilatation, which may have a role in the beneficial effects of STS. Although $\mathrm{H}_{2} \mathrm{~S}$ was reported to inhibit the activity of renin [25], we found that STS had little effect on its expression in this model. Collectively, these findings suggest that rebalance of the RAS might be involved in the protective effects of STS in our model. 
There are still some limitations. One limitation of this study is that we did not analyze other organs that control BP. The protective effects of STS against hypertension may be attributed to other organ systems, such as the vasculature, the heart, or the brain. For future research, it would be interesting to conduct a thorough examination of aforementioned protective mechanisms of STS in an organ-specific manner. Second, we did not determine which cells in the kidney are involved in the expression of $\mathrm{H}_{2} \mathrm{~S}$-generating enzymes by immunohistochemistry. Another limitation is that we did not test other treatment doses or duration of STS. Whether STS treatment displays a dose- or time-dependent effect deserves further clarification. Although the use of STS has been approved for the treatment of calciphylaxis in patients with CKD [26], its safety and efficacy for hypertension await further evaluation prior to clinical translation. Lastly, we should notice that aforementioned mechanisms might not cover the whole picture of the protective benefits of STS treatment. Considering that gut microbiota are another source of $\mathrm{H}_{2} \mathrm{~S}$ [17], additional studies are required to illuminate whether the gut microbiota are also involved in the protective role of STS against hypertension.

\section{Conclusions}

In conclusion, we demonstrated that the oral administration of STS improves hypertension in an adenine-induced CKD model, which brings us closer to clinical translation. Approaches targeting the $\mathrm{H}_{2} \mathrm{~S}$ pathway might have therapeutic potential in CKD-induced hypertension.

Author Contributions: Conceptualization, C.-N.H. and Y.-L.T.; data curation, C.-N.H., C.-Y.H., H.W.Y., G.-P.C.-C., S.L. and Y.-L.T.; funding acquisition, Y.-L.T.; project administration, C.-N.H., H.-W.Y. and Y.-L.T.; writing—original draft, C.-N.H. and Y.-L.T.; writing—review and editing, C.-N.H., H.W.Y., C.-Y.H., G.-P.C.-C., S.L. and Y.-L.T. All authors have read and agreed to the published version of the manuscript.

Funding: This work was supported by grant CORPG8L0121 from the Kaohsiung Chang Gung Memorial Hospital, Kaohsiung, Taiwan, and National Sun Yat-sen University, Kaohsiung, Taiwan.

Institutional Review Board Statement: All animal studies were approved by the Institutional Animal Ethics Committee (IACUC) of Chang Gung Memorial Hospital (Permit Number 2020073101).

Informed Consent Statement: Not applicable.

Data Availability Statement: Data are contained within the article.

Acknowledgments: We would like to thank the Center for Environmental Toxin and Emerging Contaminant Research and the Super Micro Mass Research and Technology Center, Cheng Shiu University, Kaohsiung for technical support.

Conflicts of Interest: The authors declare no conflict of interest.

\section{References}

1. Kimura, H. The physiological role of hydrogen sulfide and beyond. Nitric Oxide 2014, 41, 4-10. [CrossRef] [PubMed]

2. Feliers, D.; Lee, H.J.; Kasinath, B.S. Hydrogen sulfide in renal physiology and disease. Antioxid. Redox Signal. 2016, 25, 720-731. [CrossRef] [PubMed]

3. Lozano, R.; Naghavi, M.; Foreman, K.; Lim, S.; Shibuya, K.; Aboyans, V.; Abraham, J.; Adair, T.; Aggarwal, R.; Ahn, S.Y.; et al. Global and regional mortality from 235 causes of death for 20 age groups in 1990 and 2010: A systematic analysis for the Global Burden of Disease Study 2010. Lancet 2012, 380, 2095-2128. [CrossRef]

4. Scammahorn, J.J.; Nguyen, I.T.N.; Bos, E.M.; Van Goor, H.; Joles, J.A. Fighting oxidative stress with sulfur: Hydrogen sulfide in the renal and cardiovascular systems. Antioxidants 2021, 10, 373. [CrossRef]

5. Wilcox, C.S. Oxidative stress and nitric oxide deficiency in the kidney: A critical link to hypertension? Am. J. Physiol. Regul. Integr. Comp. Physiol. 2005, 289, R913-R935. [CrossRef]

6. Hsu, C.N.; Tain, Y.L. Gasotransmitters for the therapeutic prevention of hypertension and kidney disease. Int. J. Mol. Sci. 2021, 22, 7808. [CrossRef] [PubMed]

7. Wen, Y.D.; Wang, H.; Zhu, Y.Z. The Drug developments of hydrogen sulfide on cardiovascular disease. Oxid. Med. Cell. Longev. 2018, 2018, 1-21. [CrossRef] 
8. Hsu, C.N.; Lin, Y.J.; Lu, P.C.; Tain, Y.L. Early supplementation of D-cysteine or L-cysteine prevents hypertension and kidney damage in spontaneously hypertensive rats exposed to high-salt intake. Mol. Nutr. Food Res. 2018, 62, 2. [CrossRef]

9. Olson, K.R.; Deleon, E.R.; Gao, Y.; Hurley, K.; Sadauskas, V.; Batz, C.; Stoy, G.F. Thiosulfate: A readily accessible source of hydrogen sulfide in oxygen sensing. Am. J. Physiol. Regul. Integr. Comp. Physiol. 2013, 305, R592-R603. [CrossRef]

10. Nguyen, I.T.N.; Klooster, A.; Minnion, M.; Feelisch, M.; Verhaar, M.C.; van Goor, H.; Joles, J.A. Sodium thiosulfate improves renal function and oxygenation in L-NNA-induced hypertension in rats. Kidney Int. 2020, 98, 366-377. [CrossRef]

11. Diwan, V.; Brown, L.; Gobe, G.C. Adenine-induced chronic kidney disease in rats. Nephrology 2018, 23, 5-11. [CrossRef]

12. Hsu, C.N.; Yang, H.W.; Hou, C.Y.; Chang-Chien, G.P.; Lin, S.; Tain, Y.L. Maternal adenine-induced chronic kidney disease programs hypertension in adult male rat offspring: Implications of nitric oxide and gut microbiome derived metabolites. Int. J. Mol. Sci. 2020, 21, 7237. [CrossRef] [PubMed]

13. Hsu, C.N.; Hou, C.Y.; Chang-Chien, G.P.; Lin, S.; Tain, Y.L. Maternal Garlic Oil Supplementation Prevents High-Fat Diet-Induced Hypertension in Adult Rat Offspring: Implications of $\mathrm{H}_{2} \mathrm{~S}-$ Generating Pathway in the Gut and Kidneys. Mol. Nutr. Food Res. 2021, 65, e2001116. [CrossRef] [PubMed]

14. Bode-Böger, S.M.; Scalera, F.; Ignarro, L.J. The L-arginine paradox: Importance of the L-arginine/asymmetrical dimethylarginine ratio. Pharmacol. Ther. 2007, 114, 295-306. [CrossRef]

15. Dugbartey, G.J. The smell of renal protection against chronic kidney disease: Hydrogen sulfide offers a potential stinky remedy. Pharm. Rep. 2018, 70, 196-205. [CrossRef] [PubMed]

16. Lin, S.; Visram, F.; Liu, W.; Haig, A.; Jiang, J.; Mok, A.; Lian, D.; Wood, M.E.; Torregrossa, R.; Whiteman, M.; et al. GYY4137, a slow-releasing hydrogen sulfide donor, ameliorates renal damage associated with chronic obstructive uropathy. J. Urol. 2016, 196, 1778-1787. [CrossRef] [PubMed]

17. Hsu, C.N.; Tain, Y.L. Preventing Developmental Origins of Cardiovascular Disease: Hydrogen Sulfide as a Potential Target? Antioxidants 2021, 10, 247. [CrossRef] [PubMed]

18. Hsu, C.N.; Hou, C.Y.; Chang-Chien, G.P.; Lin, S.; Tain, Y.L. Maternal N-Acetylcysteine Therapy Prevents Hypertension in Spontaneously Hypertensive Rat Offspring: Implications of Hydrogen Sulfide-Generating Pathway and Gut Microbiota. Antioxidants 2020, 9, 856. [CrossRef] [PubMed]

19. Li, Z.; Polhemus, D.J.; Lefer, D.J. Evolution of Hydrogen Sulfide Therapeutics to Treat Cardiovascular Disease. Circ. Res. 2018, 123, 590-600. [CrossRef] [PubMed]

20. Cicone, J.S.; Petronis, J.B.; Embert, C.D.; Spector, D.A. Successful treatment of calciphylaxis with intravenous sodium thiosulfate Am. J. Kidney Dis. 2004, 43, 1104-1108. [CrossRef]

21. Zhang, X.; Yu, Y.; Lei, H.; Cai, Y.; Shen, J.; Zhu, P.; He, Q.; Zhao, M. The Nrf-2/HO-1 Signaling Axis: A Ray of Hope in Cardiovascular Diseases. Cardiol. Res. Pract. 2020, 2020, 5695723. [CrossRef] [PubMed]

22. Lee, K.S.; Kim, J.; Kwak, S.N.; Lee, K.S.; Lee, D.K.; Ha, K.S.; Won, M.H.; Jeoung, D.; Lee, H.; Kwon, Y.G.; et al. Functional role of NF- $\mathrm{KB}$ in expression of human endothelial nitric oxide synthase. Biochem. Biophys. Res. Commun. 2014, 448, 101-107. [CrossRef] [PubMed]

23. Siragy, H.M.; Carey, R.M. Role of the intrarenal renin-angiotensin-aldosterone system in chronic kidney disease. Am. J. Nephrol. 2010, 31, 541-550. [CrossRef] [PubMed]

24. Forrester, S.J.; Booz, G.W.; Sigmund, C.D.; Coffman, T.M.; Kawai, T.; Rizzo, V.; Scalia, R.; Eguchi, S. Angiotensin II Signal Transduction: An Update on Mechanisms of Physiology and Pathophysiology. Physiol. Rev. 2018, 98, 1627-1738. [CrossRef] [PubMed]

25. Lu, M.; Liu, Y.-H.; Goh, H.S.; Wang, J.J.X.; Yong, Q.-C.; Wang, R.; Bian, J.-S. Hydrogen Sulfide Inhibits Plasma Renin Activity. J. Am. Soc. Nephrol. 2010, 21, 993-1002. [CrossRef] [PubMed]

26. Peng, T.; Zhuo, L.; Wang, Y.; Jun, M.; Li, G.; Wang, L.; Hong, D. Systematic review of sodium thiosulfate in treating calciphylaxis in chronic kidney disease patients. Nephrology 2018, 23, 669-675. [CrossRef] 